\title{
SUFFICIENT CONDITIONS FOR A BUNDLE-LIKE FOLIATION TO ADMIT A RIEMANNIAN SUBMERSION ONTO ITS LEAF SPACE
}

\author{
RICHARD H. ESCOBALES, JR.
}

\section{To Bill Huebsch}

\begin{abstract}
This note furnishes a necessary and sufficient condition for a bundlelike foliation to be defined globally by a Riemannian submersion.
\end{abstract}

Introduction. The purpose of this note is to furnish sufficient conditions for a foliated manifold with a bundle-like metric to admit a Riemannian submersion onto its leaf space in a natural way. The main result, Theorem 2.2, says that this will occur whenever all the leaves are closed and the holonomy of each leaf, with respect to the foliation, is trivial. Since a foliation with a bundle-like metric can be thought of as one being defined locally by Riemannian submersions, this note provides, on the basis of a result of Hermann, a necessary and sufficient condition for the foliation to be defined globally by a Riemannian submersion. Several applications of the result to recover known theorems conclude the paper.

This work was done while the author was on sabbatical from Canisius College. $\mathrm{He}$ is grateful to the college for that opportunity. He is grateful to Professors Graver and Church of Syracuse University for their hospitality while in Syracuse, to Professor Blumenthal for some reprints, and to Professors Boothby, Conlon and Jensen who organized an NSF regional conference on foliations at Washington University.

1. Let $M$ be a $C^{\infty}$ differentiable (Hausdorff) manifold which throughout this paper is assumed to be connected and complete. Assume $M$ has a codimension $q$ foliation which is denoted by $\mathcal{V}$. Then this foliation may be defined by a maximal family of $C^{\infty}$ submersions, $f_{\alpha}: U_{\alpha} \rightarrow f_{\alpha}\left(U_{\alpha}\right) \subset R^{q}$, where $\left\{U_{\alpha}\right\}_{\alpha \in \Lambda}$ is an open cover of $M$ and where for each $p \in U_{\alpha} \cap U_{\beta}$, there is some local $C^{\infty}$ diffeomorphism, $\phi_{\beta \alpha}^{p}$, of $R^{q}$ so that $f_{\beta}=\phi_{\beta \alpha}^{p} \circ f_{\alpha}$ in some neighborhood $U_{p}$ of $p . U_{p}$ may be chosen so it is in $U_{\alpha} \cap U_{\beta}$. In fact, if $p^{\prime} \in U_{\alpha} \cap U_{\beta}$, $\phi_{\beta \alpha}^{p}=\phi_{\beta \alpha}^{p^{\prime}}$ on $f_{\alpha}\left(U_{p} \cap U_{p^{\prime}}\right)$ and $\phi_{\beta \alpha}^{p}=$ $\phi_{\beta \gamma}^{p} \circ \phi_{\gamma \alpha}^{p}$ whenever this equation makes sense (see Lawson [8, pp. 2-3]). Observe that a tangent vector $V_{p}$ belongs to the tangent space of the leaf through $p, \mathcal{T}_{p}$, if and only if $f_{\alpha *} V_{p}=0$ or $V \in \operatorname{ker} f_{\alpha_{*} p}$.

Received by the editors March 19, 1981 and, in revised form, June 4, 1981.

1980 Mathematics Subject Classification. Primary 57D30; Secondary 53C25. 
Now fix a Riemannian metric $\langle$, $\rangle$ on $M$. Then the metric $\langle$, $\rangle$ determines a distribution orthogonal to $\mathfrak{V}$ which we denote by $\mathcal{H}$. If $E$ and $F$ are arbitrary tangent vectors on $M, \mathcal{V} E$ and $\mathcal{H} E$ are the projections onto the distributions $\mathcal{V}$ and $\mathcal{H}$, respectively. If $\mathscr{V} E=E, E$ is called vertical; if $\mathcal{H} F=F, F$ is called horizontal.

Now let us restrict ourselves to $U_{\alpha}$ and consider the submersions $f_{\alpha}: U_{\alpha} \rightarrow R^{q}$ which define the foliation $\mathcal{T}$. We say a horizontal vector field $X$ on $U$ is $f_{\alpha}$ basic provided $f_{\alpha_{*}} X_{p}=f_{\alpha_{*}} X_{p^{\prime}}$ for every $p$ and $p^{\prime}$ in a connected component or plaque of $U_{\alpha} \cap L$ where $L$ is any leaf of $\mathfrak{V}$. In [3], we established the following result for horizontal vector fields. For convenience, we include its proof here.

Proposition 1.1. A horizontal vector field on $U_{\alpha} \cap U_{\beta}$ is $f_{\alpha}$ basic if and only if it is $f_{\beta}$ basic.

Proof. To prove this result, assume $X$ is $f_{\alpha}$ basic and $p$ and $p^{\prime}$ both lie in a plaque of $U_{\alpha} \cap U_{\beta} \cap L$. Using the notation above, if $p$ and $p^{\prime}$ both lie in $U_{p} \cap U_{p^{\prime}}$, then

$$
f_{\beta *} X_{p}=\phi_{\beta \alpha_{*}}^{p} f_{\alpha *} X_{p}=\phi_{\beta \alpha_{*}}^{p^{\prime}} f_{\alpha_{*}} X_{p^{\prime}}=f_{\beta *} X_{p^{\prime}}
$$

and so we are done. If $p$ and $p^{\prime}$ do not lie in $U_{p} \cap U_{p^{\prime}}$, we can choose a path in the plaque of $U_{\alpha} \cap U_{\beta} \cap L$ connecting $p$ to $p^{\prime}$ and can select for each $x$ on the path an open $U_{x} \subset U_{\alpha} \cap U_{\beta}$ so $\phi_{\beta \alpha}^{x}$ is defined on $f\left(U_{x}\right)$. Since the path is compact, the open cover $\left\{U_{x}: x \in\right.$ path $\}$ has a finite subcover, $\left\{U_{x_{i}}: 1<i<n\right\}$. If $\varepsilon$ is the Lebesgue number of the subcover, we can choose $\left\{p_{k}: 0 \leqslant k<m\right\}$ so that $p_{0}=p, p_{m}=p^{\prime}$ and $d\left(p_{i}, p_{i+1}\right)<\varepsilon$, where $d$ is the distance function on $L$ induced from the metric on $\mathcal{V}$. Then $\phi_{\beta \alpha_{*}}^{p_{i}} f_{\alpha_{*}} X_{p_{i}}=\phi_{\beta \alpha_{*}}^{p_{i+1}} f_{\alpha_{*}} X_{p_{i+1}}$ and so from (1),

$$
\begin{aligned}
f_{\beta *} X_{p} & =f_{\beta *} X_{p_{0}}=\phi_{\beta \alpha_{*}}^{p_{0}} f_{\alpha_{*}} X_{p_{0}}=\phi_{\beta \alpha_{*}}^{p_{1}} f_{\alpha_{*}} X_{p_{1}} \\
& =\cdots=\phi_{\beta \alpha_{*}}^{p_{m}} f_{\alpha_{*}} X_{p_{m}}=\phi_{\beta \alpha_{*}}^{p^{\prime}} f_{\alpha_{*}} X_{p^{\prime}}=f_{\beta_{*}} X_{p^{\prime}}
\end{aligned}
$$

We conclude $X$ is $f_{\beta}$ basic on $U_{\alpha} \cap U_{\beta}$, since $L$ was an arbitrary leaf. Switching the indices $\alpha$ and $\beta$ we obtain the converse.

2. Let $M$ be foliated as in $\S 1$ and suppose $\langle$,$\rangle is a Riemannian metric on M$. $\langle$,$\rangle is called bundle-like with respect to the foliation \mathcal{V}$ if and only if for each $\alpha$, $f_{\alpha}: U_{\alpha} \rightarrow R^{q}$ is a Riemannian submersion [10] onto its image $f_{\alpha}\left(U_{\alpha}\right)$ in $R^{q}$ or, equivalently, the metric on $\mathscr{C}$ on $U_{\alpha}$ is projectible onto its image $f_{\alpha}\left(U_{\alpha}\right) \subset R^{q}$. Notice, if $\langle$,$\rangle is bundle-like, the local diffeomorphisms, \phi_{\beta \alpha}^{p}$, of $R^{q}$ are isometries with respect to the projected metrics. In general, the metric projected onto $f_{\alpha}\left(U_{\alpha}\right)$ does not coincide with the flat metric. In [6] the following result was established.

THEOREM 2.1. (a) Let $M$ be a manifold with foliation $\mathcal{V}$ and complete Riemannian metric, $\langle$,$\rangle , that is bundle-like with respect to the foliation. Let B$ denote the set of leaves of $\mathcal{V}$ and $\pi: M \rightarrow B$ be the map: $x \rightarrow($ leaf through $x)$, for $x \in M$. Then, if all the leaves are closed in $M, B$ can be made into a metric space in such a way that $\pi$ is a continuous and open mapping that does not increase distances.

(b) If, further, the holonomy of each leaf with respect to the foliation is only the identity, then $B$ can be made into a $C^{\infty}$ manifold so that $\pi$ is a $C^{\infty}$ map of maximal rank. 
Remark 2.2. Since $B$ is a metric space, it follows $B$ is Hausdorff. We observe that in the proof of Theorem 2.1, Hermann establishes that bundle-like foliations satisfying the hypotheses (a) and (b) above are regular in the sense of Palais [11, p. 13] or simple in the sense of Haefliger [4, p. 372]. One notes that for arbitrary regular or simple foliations, the leaves are always closed [11, p. 18] and the holonomy of each leaf with respect to the foliation is trivial [4, p.379].

As a consequence of Theorem 2.1, we will establish the following result.

THEOREM 2.2. Let $M$ be a complete connected manifold with bundle-like foliation V.

(a) If all the leaves of $\mathcal{V}$ are closed and the holonomy group of each leaf with respect to the foliation is trivial, then there is a natural Riemannian metric on the leaf space $B$ so that $\pi: M \rightarrow B$ taking $x \rightarrow($ leaf through $x)$, for $x \in M$ is a Riemannian submersion.

(b) Conversely, with the notation as in (a), if $\pi: M \rightarrow B$ is a Riemannian submersion, then all the leaves are closed and each leaf has trivial foliation holonomy.

Proof. By Theorem 2.1 (a) and (b), $B$ is a $C^{\infty}$ (Hausdorff) manifold and $\pi: M \rightarrow B$ is a $C^{\infty}$ map of maximal rank. Let $L$ be any leaf of $\mathfrak{V}$. By the implicit function theorem we can find about each $p \in L$, a neighborhood $V_{p}$ of $M$ so that:

(1) Each leaf of $\mathcal{V}$ intersects $V_{p}$ in one and only one plaque or component. Observe,

(2) $V_{p} \subset U_{\alpha}$ for some $\alpha$ where the $U_{\alpha}$ are the open sets defined in $\S 1$. If $V_{p}$ were not in some $U_{\alpha}$ containing $p$, take $V_{p}^{\prime}=V_{p} \cap U_{\alpha}$ and rename $V_{p}^{\prime}, V_{p}$.

From (1), (2) and [4, §1.5, p. 372], we have

(3) If $y, z \in V_{p} \cap L^{\prime}$ where $L^{\prime}$ is any leaf of $\mathcal{V}$ with $\pi_{*} X_{y}=\pi_{*} X_{z}$ for a horizontal vector field $X$ on $U_{\alpha}$, then we also have $f_{\alpha_{*}} X_{y}=f_{\alpha_{*}} X_{z}$.

By [4, p. 372], $\pi\left(V_{p}\right)$ is an open set in $B$ and is diffeomorphic to $f_{\alpha}\left(V_{p}\right) \subset R^{q}$. Suppose $X$ is a horizontal vector field on $V_{p}$ (i.e. $X_{z}$ belongs to $\mathcal{K}_{z}$ for all $z \in V_{p}$ ) and assume $\pi_{*} X$ is a well-defined vector field on $\pi\left(V_{p}\right) \subset B$. We set

$$
\left\langle\pi_{*} X_{p}, \pi_{*} X_{p}\right\rangle_{\pi(p)} \stackrel{\text { def }}{=}\left\langle f_{\alpha_{*}} X_{p}, f_{\alpha_{*}} X_{p}\right\rangle_{f_{\alpha}(p)}
$$

Since every tangent vector $X_{\pi(p)}^{*}$ of $B$ at $\pi(p)$ can be lifted to a unique horizontal family defined on $V_{p} \cap L$, we see that (4) induces an inner product on $T_{\pi(p)} B$ using the bilinearity of $\langle$,$\rangle . Evidently, the definition does not depend on the V_{p}$ which satisfies (1) and (2).

In fact, the metric on $T_{\pi(p)} B$ does not depend on $\alpha$, since

$$
\begin{aligned}
\left\langle\pi_{*} X_{p}, \pi_{*} X_{p}\right\rangle_{\pi(p)} \stackrel{\text { def }}{=}\left\langle f_{\alpha_{*}} X_{p}, f_{\alpha_{*}} X_{p}\right\rangle_{f_{\alpha}(p)} \\
=\left\langle\phi_{\beta \alpha_{*}}^{p} f_{\alpha_{*}} X_{p}, \phi_{\beta \alpha_{*}}^{p} f_{\alpha_{*}} X_{p}\right\rangle \\
=\left\langle f_{\beta_{*}} X_{p}, f_{\beta_{*}} X_{p}\right\rangle_{f_{\beta}(p)}
\end{aligned}
$$

whenever $p \in U_{\beta}$. 
If $q \in L \cap V_{p}$, then $\pi_{*} X_{q}=\pi_{*} X_{p}=X_{\pi(p)}^{*}$, since $L \cap V_{p}$ has only one plaque in $V_{p}$. The above $V_{p}$ can be $V_{q}$ and we have $\pi(p)=\pi(q)$ and, by (3),

$$
\begin{aligned}
\left\langle\pi_{*} X_{q}, \pi_{*} X_{q}\right\rangle_{\pi(q)} & =\left\langle f_{\alpha_{*}} X_{q}, f_{\alpha_{*}} X_{q}\right\rangle_{f_{\alpha}(q)=f_{\alpha}(p)} \\
& =\left\langle f_{\alpha_{*}} X_{p}, f_{\alpha_{*}} X_{p}\right\rangle_{f_{\alpha}(p)}=\left\langle\pi_{*} X_{p}, \pi_{*} X_{p}\right\rangle_{\pi(p)} .
\end{aligned}
$$

Suppose $q \in L$ but $q \notin V_{p}$. Then there is a path $\gamma$ in $L$ connecting $p$ to $q$. For each $x \in \gamma$, there is an open set $V_{x}$ satisfying (1) and (2) so that $V_{x} \subset U_{\alpha(x)}$. Consider the cover $\left\{V_{x}\right\}$ of the segment $\gamma$ and let $\varepsilon$ be the Lebesgue number of the cover. Select a sequence $\left\{p_{i}\right\}_{0<i<m}$ so that $p_{0}=p, p_{m}=q$ and $p_{i} \in \gamma$ and $d\left(p_{i}, p_{i+1}\right)<\varepsilon$, where $d$ is the distance metric on $L$ induced from the Riemannian metric $\langle$,$\rangle . Note, p_{i}, p_{i+1}$ belong to some $V_{x}$ (and hence to some $U_{\alpha(x)}$ ). To simplify notation we denote the $V_{x}$ to which $p_{i}, p_{i+1}$ belong by $V_{i} ; U_{\alpha(x)}$ will be denoted by $U_{i}$. Thus, $p_{i+1}, p_{i+2}$ belong to $V_{i+1}$ and to $U_{i+1}$, etc. The associated submersions from $U_{i} \rightarrow R^{q}$ which define the foliation will be denoted by $f_{i}$. We will denote $X_{p_{i}}$ by $X_{i}$, where $X_{p}=X_{0}, X_{q}=X_{m}$ and $\pi_{*} X_{i}=X_{\pi(p)}^{*}$ for all $i$. Then $\pi(p)=\pi(q)$ and

$$
\begin{aligned}
\left\langle\pi_{*} X_{p}, \pi_{*} X_{p}\right\rangle_{\pi(p)} & =\left\langle f_{0 *} X_{p}, f_{0 *} X_{p}\right\rangle_{f_{0}(p)} \\
& =\left\langle f_{0 *} X_{0}, f_{0 *} X_{0}\right\rangle_{f_{0}\left(p_{0}\right)} \stackrel{\text { by (6) }}{=}\left\langle f_{0 *} X_{1}, f_{0 *} X_{1}\right\rangle_{f_{0}\left(p_{0}\right)-f_{0}\left(p_{1}\right)} \\
& \stackrel{\text { by (5) }}{=}\left\langle f_{1 *} X_{1}, f_{1 *} X_{1}\right\rangle_{f_{1}\left(p_{1}\right)} \stackrel{\text { by (6) }}{=}\left\langle f_{1 *} X_{2}, f_{1 *} X_{2}\right\rangle_{f_{1}\left(p_{1}\right)-f_{1}\left(p_{2}\right)} \\
& \stackrel{\text { by (5) }}{=}\left\langle f_{2 *} X_{2}, f_{2 *} X_{2}\right\rangle_{f_{2}\left(p_{2}\right)}=\cdots=\left\langle\pi_{*} X_{q}, \pi_{*} X_{q}\right\rangle_{\pi(q)}
\end{aligned}
$$

We conclude that the metric induced on $T_{\pi(p)} B$ is independent of the point in $L=\pi^{-1}(\pi(p))$ and so the metric on the horizontal distribution $\mathcal{H}$ induces a metric on $T B$. Since everything is evidently $C^{\infty}$ and since $\phi_{\beta \alpha}^{p}=\phi_{\beta \alpha}^{p^{\prime}}$ for $p$ and $p^{\prime}$ close, as was mentioned in $\S 1$, the projected metric is a $C^{\infty}$ metric on $B$. This proves part (a) of Theorem 2.2.

The proof of part (b) of Theorem 2.2 runs as follows: Since $\pi: M \rightarrow B$ is a Riemannian submersion the foliation is regular in the sense of Palais [11]. By [4, p. 379], the holonomy of each leaf is trivial.

REMARK 2.3. Finer results can be obtained when $\mathcal{V}$ is a Riemannian homogeneous foliation [1].

As an application of Theorem 2.2 we have the following result of Reinhart [12]. For simplicity we assume $M$ is connected and complete throughout the rest of this section.

COROLlary 2.4. Suppose the bundle-like foliation on $M$ is regular. Then, $\pi$ : $M \rightarrow B$ is a fiber space.

Proof. Since the foliation is regular, all the leaves are closed [11, p. 18] and the holonomy of each leaf is trivial. Hence, $\pi: M \rightarrow B$ is a Riemannian submersion by Theorem 2.2. That it is a fiber space follows from a result of Hermann [6] or Nagano [9]. 
COROLlary 2.5. Suppose the bundle-like foliation on $M$ is regular and the leaves are totally geodesic. Then, $\pi: M \rightarrow B$ is a fiber bundle with structure group the Lie group of isometries of the fiber.

Proof. By Theorem 2.2, $\pi: M \rightarrow B$ is a Riemannian submersion whose fibers are totally geodesic. By another result of $[6,9], \pi: M \rightarrow B$ is a fiber bundle with the indicated structure group.

REMARK 2.6. By a remark of Reinhart [12, pp. 121-122], any fiber space can be made into a Riemannian submersion. Conversely, the above cited result of Nagano and Hermann imply that any Riemannian submersion is a fiber space. Thus, in the $C^{\infty}$ case, Riemannian submersions and fiber spaces are equivalent. What Theorem 2.2 adds is that the metric on the leaf space is naturally compatible with the bundle-like metric.

\section{REFERENCES}

1. R. Blumenthal, Riemannian homogeneous foliations without holonomy (preprint).

2. C. Ehresmann, Les connexions infinitesmals, Colloq. Topologie (Espaces Fibres), Bruxelles, 1950, pp. 29-95.

3. Richard H. Escobales, Jr., The integrability tensor for bundle-like foliations, Trans. Amer. Math. Soc. (to appear).

4. A. Haefliger, Variétés feuilletées, Ann. Scuola Norm Sup. Pisa (3) 16 (1962), 367-379.

5. R. Hermann, A sufficient condition that a map of Riemannian manifolds be a fiber bundle, Proc. Amer. Math. Soc. 11 (1960), 236-242.

6. $\ldots$ On the differential geometry of foliations, Ann. of Math. (2) 72 (1960), 445-457.

7. H. Blaine Lawson, Jr., Foliations, Bull. Amer. Math. Soc. 80 (1974), 369-418.

8. _ The quantitative theory of foliations, CBMS Reg. Conf. Ser. in Math., vol. 27, Amer. Math. Soc. Providence, R. I., 1977. 17-27.

9. T. Nagano, On fibred Riemann manifolds, Sci. Papers College Gen. Ed. Univ. Tokyo 10 (1960),

10. B. O'Neill, The fundamental equations of a submersion, Michigan Math. J. 13 (1966), 459-469.

11. R. Palais, A global formulation of the Lie theory of transformation groups, Mem. Amer. Math. Soc. No. 22 (1957).

12. B. L. Reinhart, Foliated manifolds with bundle-like metrics, Ann. of Math. (2) 69 (1959), 119-131.

Department of Mathematics, Canisius College, Buffalo, New York 14208 